\title{
Autonomia em procedimentos ventilatórios por fisioterapeutas que atuam em fisioterapia intensiva no estado da Bahia: um estudo transversal
}

\author{
Autonomy in ventilatory procedures by \\ physiotherapists working in the intensive care in the \\ state of Bahia: a cross-sectional study
}

\author{
Luis Artur Santiago dos Santos ${ }^{1}$ (]) \\ Bruno Prata Martinez 2 (1) \\ Marcele Barbosa Ferreira ${ }^{3}$ (1)
}

\author{
Flávia Maria Veloso Silva ${ }^{4}$ (1) \\ Queila Ferraz Pixitelli5 \\ Helena França Correia ${ }^{6}$
}

1-3Universidade Federal da Bahia (Salvador). Bahia, Brasil. luis_santiago_3@hotmail.com, brunopmartinez@hotmail.com, fisio.marcelebf@outlook.com
455Escola Bahiana de Medicina e Saúde Pública (Salvador). Bahia, Brasil. flavia.veloso@hotmail.com, queilaferraz17@hotmail.com
${ }^{6}$ Autora para correspondência. Universidade Federal da Bahia (Salvador). Bahia, Brasil. lenafrancorreia@gmail.com

RESUMO | INTRODUÇÃo: A Unidade de Terapia Intensiva (UTI) é um local destinado ao suporte adequado para pacientes que requerem monitorização e cuidado constante. Neste ambiente o fisioterapeuta auxilia na manutenção de funções vitais e colabora para a redução de complicações clínicas e do índice de mortalidade. Além disso, dentro das suas áreas de domínio, o fisioterapeuta compartilha a responsabilidade do manejo de procedimentos ventilatórios que substituem a ventilação espontânea. OBJETIVO: Descrever a autonomia em procedimentos ventilatórios pelos fisioterapeutas que atuam em UTI no estado da Bahia. METODOLOGIA: Trata-se de um estudo transversal com fisioterapeutas que atuam em UTI no estado da Bahia, inscritos no Conselho Regional de Fisioterapia e Terapia Ocupacional da $7^{a}$ Região (CREFITO-7), utilizando um questionário eletrônico desenvolvido pelos pesquisadores. Os dados foram submetidos à análise estatística descritiva e multivariada. O nível de significância adotado foi de $p<$ 0,05. O tratamento estatístico foi realizado utilizando-se o Statistical Package for the Social Sciences, versão 21.0 (SPSS Inc., Chicago, IL, EUA). RESULTADOS: Foram avaliados 265 fisioterapeutas que atuam em terapia intensiva no estado da Bahia, com média de idade de 32,4 $\pm 5,4$ anos, sendo $61,9 \%$ do sexo feminino. Em relação a autonomia profissional, $94,3 \%$ declararam que a tomada de decisão (sobre os procedimentos fisioterapêuticos na UTI em que atuam) é de responsabilidade do fisioterapeuta. O maior nível de autonomia sobre os procedimentos ventilatórios foi observado para a aplicação de Ventilação Mecânica Não Invasiva VNI $(97,7 \%)$, seguido do desmame $(97,4 \%)$, indicação (97\%) e manutenção $(96,2 \%)$. CONCLUSÃo: Através do presente estudo foi possível concluir que os fisioterapeutas que atuam em UTI no Estado da Bahia declaram possuir autonomia profissional em relação a procedimentos ventilatórios, sobretudo para os não invasivos.

PALAVRAS-CHAVE: Autonomia profissional. Fisioterapia. Unidade de Terapia Intensiva. Serviço hospitalar de fisioterapia. Suporte ventilatório invasivo. Ventilação não invasiva.
ABSTRACT | INTRODUCTION: The Intensive Care Unit (ICU) is a ward intended to the specialized support to critically ill patients or after undergoing a highly complex procedure, who need constant monitoring and care. In this environment, the physiotherapist works to maintain vital functions and helps reduce clinical complications and mortality rates. Furthermore, within their domains, the physiotherapist shares the responsibility for managing methods that replace spontaneous breaths. OBJECTIVE: To describe the autonomy in ventilatory procedures by physiotherapists working in ICUs in the state of Bahia. METHODOLOGY: This is a cross-sectional study with physiotherapists working in ICUs in the state of Bahia, registered at the Regional Council of Physiotherapy and Occupational Therapy of the 7th Region (CREFITO-7). In data, collect was used an electronic questionnaire was developed by the researchers. The data collected was analyzed through descriptive and multivariate statistics. A p-value $<0.05$ was set as statistically significant. Statistical analysis was performed with Statistical Package for the Social Sciences, 21.0 version (SPSS Inc., Chicago, IL, EUA). RESULTS: Were evaluated a total of two hundred and sixty-five (265) physiotherapists who work at an Intensive Care Unit in the state of Bahia, with a mean age of $32.4 \pm 5.4$ years, being $61.9 \%$ female. Regarding professional autonomy, $94.3 \%$ declared that decision-making about physical therapy procedures in the ICU where they work is the responsibility of the physiotherapists. The highest level of autonomy over ventilatory procedures was observed for the application of non-invasive ventilation (97.7\%), followed by weaning from mechanical ventilation (97.4\%), indication (97\%), and maintenance (96.2\%). CONCLUSION: Through this study, it was possible to conclude that physiotherapists working in ICUs in the State of Bahia claim to have professional autonomy in relation to ventilatory procedures, especially for the non-invasive ones.

KEYWORDS: Professional autonomy. Physiotherapy. Intensive care unit. Physiotherapy hospital service. Invasive ventilatory support. No invasive ventilation. 


\section{Introdução}

A Unidade de Terapia Intensiva (UTI) é local destinado para o suporte adequado de pacientes em estado grave ou após a realização de um procedimento de alta complexidade, que necessitam de monitorização e cuidado constante. Nesse sentido, as UTIs podem ser gerais (ou destinadas a casos específicos), médicas ou cirúrgicas e são organizadas por sistemas, patologias, demandas específicas ou por faixa etária. Dessa forma, podem ser divididas em unidades para adultos, pediátrica ou neonatal e acolhem pacientes com problemas neurológicos, de queimaduras ou traumas. ${ }^{1}$

Em 2010 a Resolução - RDC N07/2010 da Agência Nacional de Vigilância Sanitária (ANVISA) estabeleceu as condições necessárias para o funcionamento das UTIs e regulamentou a presença do fisioterapeuta nessa unidade, reforçando a necessidade de um responsável técnico por essas unidades sendo que os responsáveis técnicos devem ser especialistas em terapia intensiva, fisioterapia pneumofuncional ou cardiologia. ${ }^{2}$

No ano seguinte, o Plenário do Conselho Federal de Fisioterapia e Terapia Ocupacional (COFFITO) aprovou a Resolução $N^{\circ}$ 402/2011, que considerando a ética profissional do fisioterapeuta, reconhece a atividade do fisioterapeuta no exercício da especialidade de fisioterapia em Terapia Intensiva e regulamenta a atuação do fisioterapeuta intensivista em todos os níveis de atenção à saúde. $\underline{3}$ Neste ambiente, o fisioterapeuta exerce um papel relevante em benefício dos pacientes, pois auxilia na manutenção de funções vitais e colabora para a redução de complicações clínicas e do índice de mortalidade. Além disso, dentro das suas áreas de domínio, o fisioterapeuta compartilha a responsabilidade do manejo de métodos que substituem a ventilação espontânea. ${ }^{4}$

Em se tratando de condutas que substituem a ventilação espontânea no ambiente da terapia intensiva, estão dispostas a ventilação mecânica invasiva (VM) e a ventilação mecânica não invasiva (VNI). Os fisioterapeutas que atuam nessa área necessitam ser especializados e qualificados para aplicar técnicas fisioterapêuticas, para atuar tanto com a VM quanto com a VNI. Observa-se que o fisioterapeuta apresenta uma autonomia maior no manuseio da VNI. $\frac{5}{}$ Vários fatores influenciam na eficiência da técnica de VNI, como a patologia apresentada pelo paciente e o tratamento que é escolhido, mas é notável que a atuação do fisioterapeuta melhora o prognóstico dos pacientes que fazem uso da técnica. $4, \underline{6}$

Em relação à autonomia profissional, existem dados de que ela está relacionada com as expectativas individuais dos profissionais, sendo que, a falta de autonomia costuma aumentar o desgaste emocional e influenciar nas consequências negativas sofridas no ambiente de trabalho. Ter autonomia, por sua vez, possibilita o aumento da satisfação, a melhoria na prestação de cuidados e no compromisso com o atendimento do paciente. ${ }^{?}$

Sendo assim, os profissionais da área da saúde precisam ter autonomia profissional para definir condutas de aspectos positivos no trabalho, tomar decisões para efetuar procedimentos com eficiência e para garantir a segurança do atendimento prestado, oferecendo assim a atenção necessária à saúde da população. $\underline{8}$

Apesar da importância da autonomia profissional ser reconhecida e de haver uma quantidade considerável de estudos em relação ao grau de autonomia em outras classes na área da saúde, existem poucos estudos e dados sobre a autonomia do fisioterapeuta que também é um profissional pleno dessa área. Por esse motivo, é necessário realizar estudos que abordem a autonomia profissional dos fisioterapeutas em procedimentos da fisioterapia, para conhecimento do atual nível e desenvolvimento de estratégias para otimização dela, ocasionando padronização em técnicas fisioterapêuticas que resulte numa assistência de qualidade ao paciente. Sendo assim, o objetivo desse estudo é verificar o nível de autonomia em procedimentos ventilatórios e verificar os fatores associados a autonomia na indicação e desmame de procedimentos ventilatórios não invasivos pelos fisioterapeutas que atuam em UTI no estado da Bahia.

\section{Métodos}

Trata-se de um estudo transversal que pesquisou a autonomia profissional em procedimentos ventilatórios não invasivos pelos fisioterapeutas que atuam em UTI de hospitais públicos e privados no estado da Bahia, entre julho e novembro de 2018. 
Para acessar os dados foi criado um link desenvolvido na plataforma Google Forms contendo um questionário (via mala direta pelo Conselho Regional de Fisioterapia da $7^{a}$ Região) e enviado a todos os fisioterapeutas inscritos no conselho. A primeira questão foi referente a área de especialidade do profissional, aqueles que não atuavam em UTI foram direcionados a uma página de agradecimentos e excluídos da pesquisa. Esse procedimento se fez necessário diante da impossibilidade do envio do link, por parte do conselho, exclusivamente para os fisioterapeutas que atuam em UTI. A fim de garantir a confidencialidade e sigilo dos dados pessoais, o profissional respondeu o questionário, e este foi enviado aos pesquisadores apenas com as respostas. Apesar do envio via CREFITO-7 (Conselho Regional de Fisioterapia da $7^{a}$ Região), a pesquisa não tem qualquer relação com o referido conselho, e a participação foi voluntária.

O questionário elaborado pelos pesquisadores versou sobre dados sociodemográficos, formação acadêmica e profissional, autonomia profissional, contendo também questões de múltipla escolha e descritivas. Para análise da autonomia em procedimentos ventilatórios foi realizada dicotomização dessa variável, onde foi considerado como tendo autonomia para o procedimento o fisioterapeuta que declarou possuir autonomia ou autonomia mediante protocolo na indicação, aplicação, manutenção e desmame do procedimento.

A pesquisa foi aprovada pelo Comitê de Ética em Pesquisa do Instituto de Ciências da Saúde da Universidade Federal da Bahia, sob o Parecer de número: 2.642.961 em 08 de maio de 2018. Todos os esclarecimentos e os possíveis riscos foram explicados no TCLE que foi enviado juntamente com o questionário.

As variáveis categóricas foram expressas em frequências absolutas e relativas. As variáveis numéricas foram expressas em média e desvio padrão ou mediana e intervalo interquartílico, quando apropriado. O teste do qui-quadrado foi utilizado para a comparação das variáveis categóricas e, quando inadequado, o teste exato de Fisher foi utilizado. $O$ teste $t$ de Student foi utilizado para estabelecer a significância estatística da diferença entre as médias dos grupos. Após a análise univariada, as variáveis independentes foram inseridas no modelo logístico caso apresentassem um $p \leq 0,10$, permanecendo no modelo caso continuassem significantes $(p<0,05)$. Adotou-se o procedimento manual para inserção e retirada das variáveis. O nível de significância adotado foi de $p<$ 0,05 . O tratamento estatístico foi realizado utilizando-se o Statistical Package for the Social Sciences, versão 21.0 (SPSS Inc., Chicago, IL, EUA).

\section{Resultados}

Dentre os questionários enviados, 294 fisioterapeutas responderam. Entre eles, 29 declararam não atuar em terapia intensiva, resultando em 265 (90,1\%) fisioterapeutas que atuam em terapia intensiva no estado da Bahia entre julho e novembro de 2018. A média de idade dos participantes foi $32,4 \pm 5,4$ anos, com predomínio do sexo feminino (164/61,9\%), com atuação na capital do estado (174/65,7\%), em hospital público (181/68,3\%) e com tempo médio de atuação em UTI de $6,6 \pm 4,9$ anos.

Ao abordar a autonomia profissional dos entrevistados, 94,3\% (250) declararam que a tomada de decisão sobre os procedimentos fisioterapêuticos na UTI em que atuam é de responsabilidade do fisioterapeuta. O maior nível de autonomia sobre os procedimentos ventilatórios foi observado para a aplicação de VNI (259/97,7\%), seguida do desmame $(258 / 97,4 \%)$, indicação (257/97\%) e manutenção $(255 / 96,2 \%)$ da VNI. Os dados referentes a comparação entre os fisioterapeutas que atuam em UTI no estado da Bahia com e sem autonomia para indicação e desmame de procedimentos ventilatórios não invasivos estão descriminados na Tabela 1. Após a análise univariada, as variáveis participação em eventos e número de pacientes por turno foram inseridas em um modelo de regressão logística e não permaneceram associadas a autonomia para a indicação de VNI. As variáveis idade e tempo de formado não permaneceram associadas a autonomia no desmame da VNI após análise multivariada. 
Tabela 1. Comparação entre os fisioterapeutas que atuam em unidade de terapia intensiva no Estado da Bahia com e sem autonomia para indicação e desmame de procedimentos ventilatórios não invasivos entre julho e novembro de 2018

\begin{tabular}{|c|c|c|c|}
\hline \multicolumn{4}{|c|}{ AUTONOMIA NA INDICAÇÃO DE VNI } \\
\hline & SIM (257/97\%) & NÃO (8/3\%) & $\mathbf{p}$ \\
\hline Sexo feminino & $158(96,3 \%)$ & $6(76 \%)$ & 0,71 * \\
\hline Idade $(X \pm D P)$ & $38,4 \pm 5,4$ & $33 \pm 4,3$ & $0,70 \dagger$ \\
\hline Tempo em UTI & $6,62 \pm 5$ & $5,63 \pm 3,1$ & $0,40 \dagger$ \\
\hline Residência profissional & $46(17,9 \%)$ & $1(12,5 \%)$ & $1,0 *$ \\
\hline Participação em eventos & $195(76,2 \%)$ & $4(50 \%)$ & $0,10 * *$ \\
\hline Leitura de artigos & $209(81,3 \%)$ & $6(75 \%)$ & $0,65^{* *}$ \\
\hline Publicação de artigos & $71(27,8 \%)$ & $3(37,5 \%)$ & $0,11 * *$ \\
\hline Associados (AMIB/ASSOBRAFIR) & $60(23,3 \%)$ & 0 & NA \\
\hline Tempo de formado $(X \pm D P)$ & $8,75 \pm 5,2$ & $8,13 \pm 3,0$ & $0,73 \dagger$ \\
\hline Pós-graduação Strictu Sensu & $24(9,3 \%)$ & $1(12,5 \%)$ & $0,55^{* *}$ \\
\hline Instituição Pública & $48(18,7 \%)$ & $1(12,5 \%)$ & $1,0^{*}$ \\
\hline Instituição Privada & $209(81,3 \%)$ & $7(87,5 \%)$ & \\
\hline Pós em área hospitalar & $191(74,3 \%)$ & $8(100 \%)$ & NA \\
\hline Pacientes por turno de $6 \mathrm{~h}(\mathrm{X} \pm \mathrm{DP})$ & $9,27 \pm 2,2$ & $11,4 \pm 4$ & $0,01 \dagger$ \\
\hline Carga horária semanal $(X \pm D P)$ & $44 \pm 16,1$ & $37,8 \pm 11,6$ & $0,28 t$ \\
\hline \multicolumn{4}{|c|}{ AUTONOMIA NO DESMAME DE VNI } \\
\hline & SIM $258(97,4 \%)$ & NÃO $7(2,6 \%)$ & $\mathbf{p}$ \\
\hline Sexo feminino & $6(85,7 \%)$ & $158(61,3 \%)$ & $0,25 *$ \\
\hline Idade $(X \pm D P)$ & $32,3 \pm 5,4$ & $36,3 \pm 4,8$ & $0,05 t$ \\
\hline 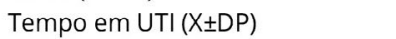 & $6,52 \pm 4,9$ & $9,29 \pm 5,2$ & $0,14 \dagger$ \\
\hline Residência profissional & $47(18,2 \%)$ & 0 & NA \\
\hline Participação em eventos & $195(75,9 \%)$ & $4(37,1 \%)$ & $0,38 * *$ \\
\hline Leitura de artigos & $210(81,4 \%)$ & $5(71,4 \%)$ & $0,62^{* *}$ \\
\hline Publicação de artigos & $73(28,3 \%)$ & $1(14,3 \%)$ & $0,69 * *$ \\
\hline Associados (AMIB/ASSOBRAFIR) & $59(22,9 \%)$ & $1(14,3 \%)$ & $1,00 * *$ \\
\hline Tempo de formado $(X \pm D P)$ & $8,62 \pm 5,2$ & $13 \pm 4,12$ & $0,03 t$ \\
\hline Pós-graduação Strictu Sensu & $24(9,3 \%)$ & $1(14,3 \%)$ & $0,50 * *$ \\
\hline Instituição Pública & $48(18,6 \%)$ & $1(14,3 \%)$ & $1,0 *$ \\
\hline Instituição Privada & $210(81,4 \%)$ & $6(85,7 \%)$ & \\
\hline Pós em área hospitalar & $193(74,8 \%)$ & $6(85,7 \%)$ & $0,68^{* *}$ \\
\hline Pacientes por turno de $6 h(X \pm D P)$ & $9,29 \pm 2,2$ & $11 \pm 5,0$ & $0,40 \dagger$ \\
\hline Carga horária semanal $(X \pm D P)$ & $43,7 \pm 16$ & $46,4 \pm 15,9$ & $0,66 t$ \\
\hline
\end{tabular}

Em relação a procedimentos ventilatórios invasivos, o maior índice de autonomia foi referente ao desmame da (258/97,4\%), seguido de manutenção (241/94,5\%), e a menor autonomia relacionada a indicação (227/85,6\%). Os dados referentes autonomia do fisioterapeuta em relação aos procedimentos ventilatórios invasivos realizados estão descritos na Figura 1. 
Figura 1. Níveis de autonomia dos fisioterapeutas que atuam em UTI no estado da Bahia em relação a procedimentos ventilatórios invasivos

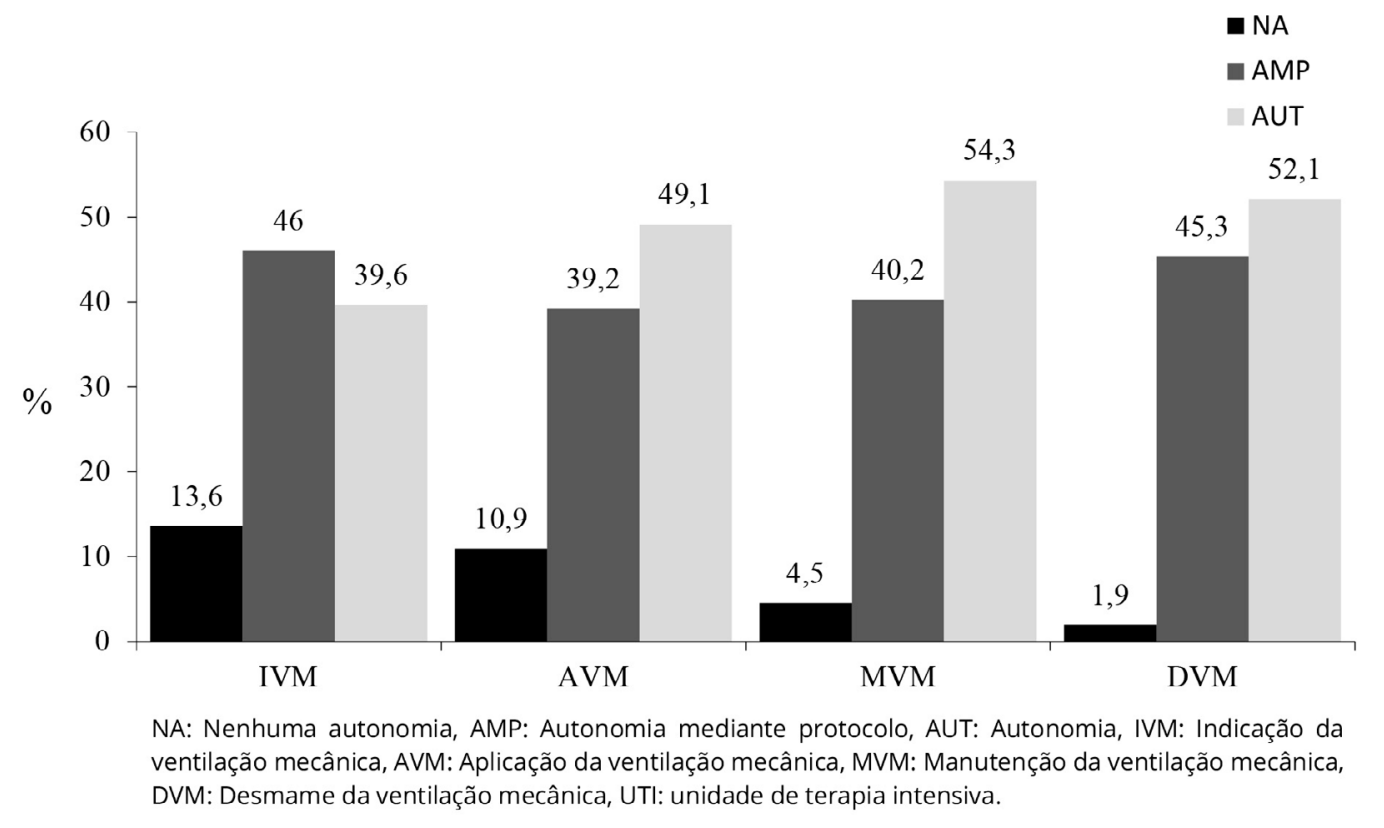

\section{Discussão}

Este estudo analisou a autonomia em procedimentos ventilatórios e os fatores associados a autonomia relacionada a procedimentos ventilatórios não invasivos dos fisioterapeutas que atuam em UTI no estado da Bahia. Referindo-se à autonomia técnica profissional, o fisioterapeuta vem buscando há anos seu aprimoramento científico. ${ }^{914}$ De acordo com estudos, a autonomia profissional tem forte ligação com o conhecimento do profissional e seus saberes ${ }^{7}$, visto que cada vez mais os profissionais frequentam os bancos dos programas de mestrado e doutorado dentro e fora do país, criando uma nova ciência, um novo pensar..$^{10,15}$

Em relação a autonomia profissional no exercício de procedimentos ventilatórios não invasivos pelos fisioterapeutas, é refletida a inserção eficiente do profissional nesse ambiente ${ }^{2,11}$, bem como a eficácia e benefício dessa terapêutica descrita em estudos anteriores no tratamento de doenças respiratórias que acometem pacientes internados no contexto da terapia intensiva. ${ }^{4,16}$ Além da autonomia profissional ser fator preponderante em relação ao desempenho profissional e satisfação no ambiente de trabalho.,12

A respeito da subdivisão das etapas da VNI em indicação, aplicação, manutenção e desmame, houve relativamente o mesmo nível de autonomia entre os pesquisados. Observando assim que apesar de serem etapas distintas, fazem parte do mesmo procedimento, conferindo habilidade do fisioterapeuta em todas as etapas da terapia, conferindo habilidade do fisioterapeuta em todas as etapas da terapia, apesar de ser uma técnica que engloba as áreas médica e de enfermagem. ${ }^{16}$

Em contrapartida, se tratando de procedimentos ventilatórios invasivos, no que diz respeito a sua indicação, aplicação, manutenção e desmame, houve uma maior quantidade de respostas declarando a falta de autonomia, ou autonomia mediante a protocolo. Fato esse que se resulta por se tratar de procedimento invasivo, que em muitas vezes o fisioterapeuta faz parte da tomada de decisão. ${ }^{17}$ Ao analisar a autonomia mediante protocolo, acredita-se em procedimentos estabelecidos de acordo a evidências relevantes sobre o assunto, trazendo uma segurança maior para sua execução, sem interferir na autonomia profissional e garantindo qualidade na assistência. 
Pesquisas realizadas online apresentam algumas limitações, a primeira delas é a dificuldade de atingir indivíduos que não possuem acesso à internet. Entretanto, acredita-se que isso não se aplique na realidade de profissionais graduados, com e-mails cadastrados em conselho profissional. A segunda é a possibilidade de baixa adesão ao questionário. Com objetivo de minimizar esse efeito buscou-se o apoio do conselho profissional regional e a sensibilização dos fisioterapeutas via redes sociais. 13,17 Outra limitação é sobre a ausência de dados, na época da aplicação dos questionários, relacionados a quantidade de Fisioterapeutas atuantes em terapia intensiva no estado da Bahia.

Este estudo pode mostrar a realidade da autonomia profissional em procedimentos ventilatórios de fisioterapeutas que atuam em UTI no estado da Bahia, podendo fornecer dados para melhoria da prática e condições de trabalho desses profissionais, visando traçar estratégias e planos para organização da categoria e melhoria também da assistência fisioterapêutica ao paciente crítico. Além disso, apontam para a importância do fisioterapeuta nesse ambiente, o que também é relatado fora da realidade brasileira. ${ }^{18}$

\section{Conclusão}

Os dados da pesquisa mostram que a maior parte dos fisioterapeutas que atuam em UTI no Estado da Bahia declara ter autonomia profissional em condutas fisioterapêuticas, e em relação a procedimentos ventilatórios há uma sobreposição na tomada de decisões relacionadas a VNI com relação a ventilação invasiva, principalmente em relação à indicação e aplicação e desmame da VNI. Variáveis sociodemográficas, de formação acadêmica e relacionadas a demanda de trabalho não estiveram associadas a autonomia profissional em relação aos procedimentos ventilatórios não invasivos.

\section{Agradecimentos}

Agradecemos ao Conselho Regional de Fisioterapia e Terapia Ocupacional da $7^{\text {a }}$ Região (CREFITO-7) pelo apoio e disponibilidade para colaborar com o andamento da pesquisa, disponibilizando o setor de tecnologia para divulgação e envio do questionário.

\section{Contribuições dos autores}

Santiago LA e Correia HF participaram da concepção, delineamento, coleta de dados da pesquisa, análise e interpretação dos resultados e redação do artigo científico. Martinez BP participou da concepção, delineamento, coleta de dados, análise e interpretação dos resultados. Ferreira MB participou da concepção, delineamento, coleta de dados e interpretação dos resultados. Silva FMV e Pixitelli, QF participaram da interpretação dos resultados e redação do artigo.

\section{Conflitos de interesses}

Nenhum conflito financeiro, legal ou político envolvendo terceiros (governo, empresas e fundações privadas etc.) foi declarado para nenhum aspecto do trabalho submetido (incluindo, mas não se limitando a subvenções e financiamentos, participação em conselho consultivo, desenho de estudo, preparação de manuscrito, análise estatística, etc.).

\section{Referências}

1. Nates JL, Nunnally M, Kleinpell R, Blosser S, Goldner J, Birriel B, et al. ICU Admission, Discharge, and Triage Guidelines: A Framework to Enhance Clinical Operations, Development of Institutional Policies, and Further Research. Crit Care Med. 2016;4(8):1553-602. https://doi.org/10.1097/ CCM. 0000000000001856

2. Ministério da Saúde (Brasil). Agência Nacional de Vigilância Sanitária. Dispõe sobre os requisitos mínimos para funcionamento de Unidades de Terapia Intensiva e dá outras providências [Internet]. Brasília: Ministério da Saúde; 2010. Disponível em: http://bvsms.saude.gov.br/bvs/saudelegis/ anvisa/2010/res0007_24_02_2010.html

3. Resolução n 402 de 03 de agosto de 2011 (Brasil). Disciplina a Especialidade Profissional Fisioterapia em Terapia Intensiva e dá outras providências [Internet]. Brasília: Conselho Federal de Fisioterapia e Terapia Ocupacional; 2011. Disponível em: http:// www.crefito3.org.br/dsn/pdfetica/Res $\% 20$ Coffito $\% 20402 \% 20-\% 20$ 03-08-2011-\%20Intensiva.pdf

4. Magalhães FLS. Os benefícios da Ventilação Não Invasiva nos pacientes internados na UTI e em ambulatórios [trabalho de conclusão de curso] [Internet]. São Francisco do Conde: Universidade da Integração Internacional da da Lusofonia AfroBrasileira; 2018. Disponível em: http://www.repositorio.unilab. edu.br:8080/xmlui/handle/123456789/724

5. Nozawa E, Sarmento GJV, Vega JM, Costa D, Silva JE, Feltrim MIZ. Perfil de fisioterapeutas brasileiros que atuam em unidades de terapia intensiva. Fisioter Pesq. 2008;15(2):177-78. https://doi. org/10.1590/S1809-29502008000200011 
6. Reis NF, Gazola NLG, Bundchen DC, Bonorino KC. Ventilação não invasiva na unidade de terapia intensiva de um hospital universitário: características relacionadas ao sucesso e insucesso. Fisioter Pesq. 2019;26(1):3-8. https://doi.org/10.1590/18092950/17000626012019

7. Bonfada MS, Moura LN, Soares SGA, Pinno C, Campogonara S. Autonomia do enfermeiro no ambiente hospitalar. Enfermagem Brasil. 2018;17(5):527-34. https://doi.org/10.33233/eb.v17i5.1503

8. Melo CMM, Florentino TC, Mascarenhas NB, Macedo KS, Silva MC, Mascarenhas SN. Autonomia profissional da enfermeira: algumas reflexões. Esc Anna Nery. 2016;20(4):e20160085. https:// doi.org/10.5935/1414-8145.20160085

9. Hodgson CL, Tipping CJ. Physiotherapy management of intensive care unit-acquired weakness. J Physiother. 2017;63(1):410. https://doi.org/10.1016/j.jphys.2016.10.011

10. Barros FBM. Autonomia Profissional do Fisioterapeuta ao longo da história. Fisiobrasil [Internet]. 2003;29:20-31. Disponível em: https://www.researchgate.net/publication/321186076. AUTONOMIA_PROFISSIONAL_DO_FISIOTERAPEUTA_AO_LONGO DA HISTORIA

11. Câmara AMSC, Santos LLCP. Um estudo com egressos do curso de fisioterapia da Universidade Federal de Minas Gerais (UFMG): 1982-2005. Rev Bras Educ Med. 2012;36(1):5-17. https:// doi.org/10.1590/S0100-55022012000200002

12. Prati FAM. Autonomia profissional do fisioterapeuta e do terapeuta ocupacional [Internet]. Porto Alegre: Conselho Federal de Fisioterapia e Terapia Ocupacional; 2016. Disponível em: https://crefito5.org.br/noticia/artigo-traz-esclarecimentos-sobreautonomia-do-fisioterapeuta-e-do-terapeuta-ocupacional
13. Rocha AR, Russo RC, Toledo TR, Rodrigues JE. Perfil de formação profissional dos fisioterapeutas das unidades de terapia intensiva da cidade de Maceió. ASSOBRAFIR Ciênc [Internet]. 2012;3(2):21-30. Disponível em: https://www.cpcrjournal.org/ article/5de11d000e8825983c4ce1d5

14. Barbas CSV, Ísola AM, Farias AMC, Cavalcanti AB, Gama AMC, Duarte ACM. Recomendações brasileiras de ventilação mecânica 2013. Rev Bras Ter Intensiva. 2013;26(2):89-121. https://doi. org/10.5935/0103-507X.20140017

15. Caetano JA, Soares E, Andrade LM, Ponte RM. Cuidado humanizado em terapia intensiva: um estudo reflexivo. Esc Anna Nery. 2007;11(2):325-30. https://doi.org/10.1590/S1414$\underline{81452007000200022}$

16. Yamanaka CS, Góis AFT, Vieira PCB, Alves JCD, Oliveira LM, Blanes $L$, et al. Intubação orotraqueal: avaliação do conhecimento médico e das práticas clínicas adotadas em unidades de terapia intensiva. Rev Bras Ter Intensiva. 2010;22(2):103-11. https://doi. org/10.1590/S0103-507X2010000200002

17. Badaró AFV, Guilhem D. Perfil sociodemográfico e profissional de fisioterapeutas e origem das suas concepções sobre ética. Fisioter Mov. 2011;24(3):445-54. https://doi.org/10.1590/S010351502011000300009

18. Stiller K. Physiotherapy in intensive care: An updated systematic review. Chest. 2013;144(3):825-47. https://doi. org/10.1378/chest.12-2930 